\title{
Hydrodynamical simulations of G2 interpreted as a diffuse gas cloud
}

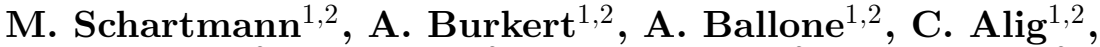 \\ S. Gillessen ${ }^{2}$, R. Genzel ${ }^{2}$, F. Eisenhauer ${ }^{2}$ and T. Fritz ${ }^{2}$ \\ ${ }^{1}$ Universitäts-Sternwarte München, Scheinerstraße 1, D-81679 München, Germany \\ email: schartmann@mpe.mpg.de \\ ${ }^{2}$ Max-Planck-Institut für extraterrestrische Physik, Postfach 1312, Giessenbachstr., D-85741 \\ Garching, Germany
}

\begin{abstract}
Recently the gas and dust cloud "G2" was discovered on a highly eccentric orbit around the massive black hole in the Galactic center. The orbit will bring the cloud as close as 2400 Schwarzschild radii to Sgr A* beginning of 2014. With the help of hydrodynamical simulations using the PLUTO code, we investigate possible origins and the fate of the cloud in the coming years. In this proceedings article, we concentrate on a scenario where G2 is interpreted as a diffuse gas cloud and show its detailed evolution in the observable positionvelocity diagrams. We further elaborate on the problem of the tail emission which might or might not be related to the G2 cloud.
\end{abstract}

Keywords. black hole physics — hydrodynamics — methods: numerical — ISM: clouds — Galaxy: center

\section{Introduction}

Recently, Gillessen et al. (2012, 2013a,b) discovered a fast moving object within the range of the S-stars close to Sgr A*. The observed fluxes in the $K$ (upper limit), $L$ and $M$ bands indicate that it is a dusty, ionized gas cloud with a dust temperature of approximately $550 \mathrm{~K}$. The data accurately constrain the orbit to be highly eccentric $(\mathrm{e}=0.98)$ with a pericenter passage at a distance of only 2400 Schwarzschild radii, which is currently ongoing. The cloud has a mass of 3 Earth masses and an orbital period of 400 years. The observed position-velocity diagrams clearly show the tidal interaction with the central massive black hole. In the 2013 observations a fraction of the cloud has already passed pericenter and shows up in the blue-shifted part of the PV diagram.

\section{The compact cloud scenario}

With the help of hydrodynamical simulations with the PLUTO code (Mignone et al., 2007) we investigate G2's origin and fate (Burkert et al., 2012 and Schartmann et al., 2012). For the simulations presented in these proceedings, we assume that the cloud started in pressure equilibrium at the 1995.5 position of the observed orbit (of Gillessen et al., 2012) in spherical shape, following a test particle analysis. As can be seen in Figure 1, the cloud stretches due to tidal forces of the central massive black hole, which also accelerates the destruction of the cloud due to the interaction with the hot ambient atmosphere by its ram pressure and Kelvin-Helmholtz instabilities. The atmosphere is treated with an idealized model based on ADAF solutions by Yuan et al. (2003). After pericenter passage, the cloud gets disrupted and feeds gas towards Sgr A* in a clumpy stream. Good agreement is found between the simulations and the observed positionvelocity diagrams, showing dramatic changes near the pericenter passage (see Figure 2). 

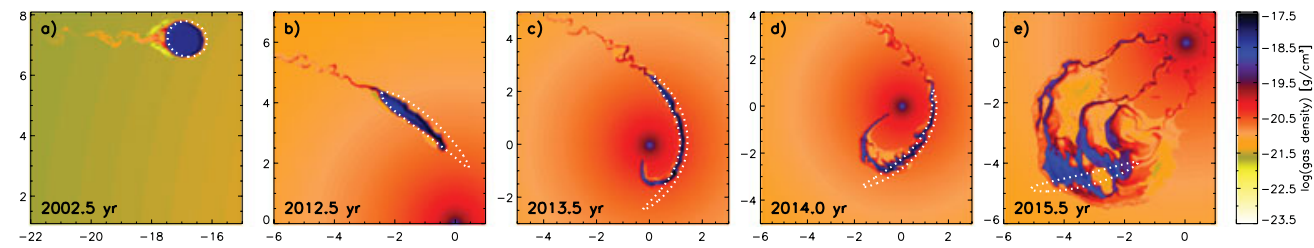

Figure 1. Density evolution of our Compact Source Scenario. White dotted lines are the result of a test particle simulation. [A COLOR VERSION IS AVAILABLE ONLINE.]
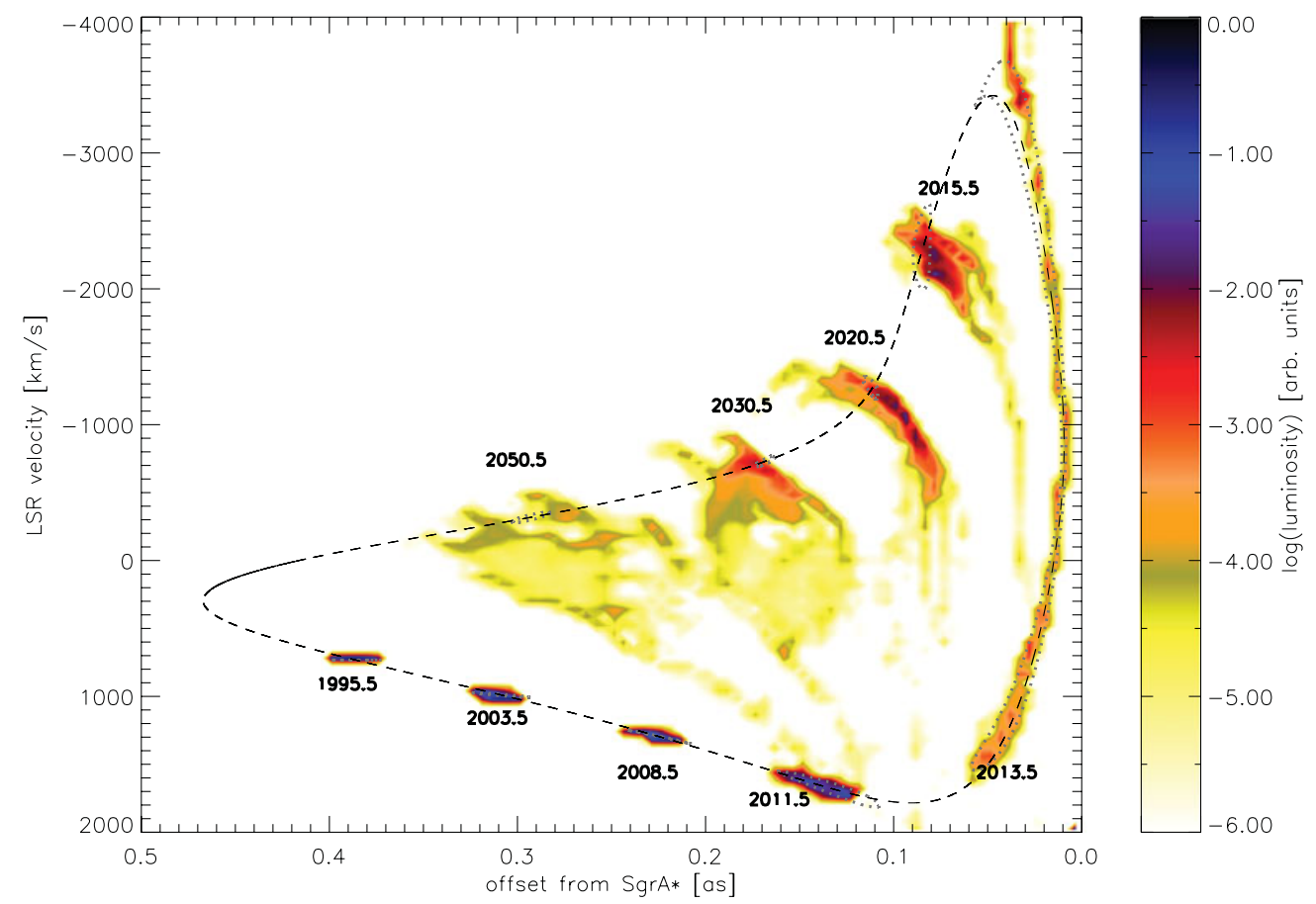

Figure 2. Evolution of the simulation in the position-velocity diagram.

[A COLOR VERSION IS AVAILABLE ONLINE.]

They also show a spreading of the cloud, which is in contrast to our expectations for the Compact Source Scenario (Ballone et al., 2013 and these proceedings), which is expected to reform the cloud after pericenter.

\section{The problem of the tail}

So far, only the main component G2 has been investigated. However, it seems to be followed by a trailing component of similar mass. The latter cannot be explained by gas stripped from a cloud caused by hydrodynamical interactions. One possible explanation could be that G2 is only the front part of a larger spherical shell on a slightly different orbit. Alternatively, both components could be part of a clumpy stream of gas pointing towards Sgr A*.

\section{References}

Ballone, A., Schartmann, M., Burkert, A., Gillessen, S., Genzel, R., Fritz, T. K., Eisenhauer, F., Pfuhl, O., \& Ott, T. 2013, ApJ 776, 13 
Burkert, A., Schartmann, M., Alig, C., Gillessen, S., Genzel, R., Fritz, T. K., \& Eisenhauer, F. 2012, ApJ 750, 58

Gillessen, S., Genzel, R., Fritz, T. K., Quataert, E., Alig, C., Burkert, A., Cuadra, J., Eisenhauer, F., Pfuhl, O., Dodds-Eden, K., Gammie, C. F., \& Ott, T. 2012, Nature 481, 51

Gillessen, S., Genzel, R., Fritz, T. K., Eisenhauer, F., Pfuhl, O., Ott, T., Cuadra, J., Schartmann, M., \& Burkert, A. 2013, ApJ 763, 78

Gillessen, S., Genzel, R., Fritz, T. K., Eisenhauer, F., Pfuhl, O., Ott, T., Schartmann, M., Ballone, A., \& Burkert, A. 2013, ApJ 774, 44

Mignone, A., Bodo, G., Massaglia, S., Matsakos, T., Tesileanu, O., Zanni, C., \& Ferrari, A. 2007, ApJS 170, 228

Schartmann, M. and Burkert, A. and Alig, C. and Gillessen, S. and Genzel, R. and Eisenhauer, F. and Fritz, T. K. 2012, ApJ 755, 155

Yuan, F., Quataert, E., \& Narayan, R. 2003, ApJ 598, 301 